\title{
Etiology of Pleural Effusion among Adults in Three University Hospitals in Beirut: A One-Year Retrospective Cross Sectional Analytical Study
}

\author{
Ali IBRAHIM, Amrou RIDA*, Ibrahim MALASS and Loubna Tayara \\ Universite Libanaise, Lebanon

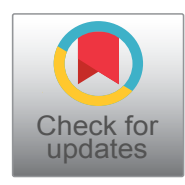

*Corresponding author: Amrou Rida, Faculty of Medical Sciences, Universite Libanaise, Lebanon

\begin{abstract}
Background: Pleural effusion, the pathological accumulation of fluid in the pleural space, is very common. It has a wide differential diagnosis, and a delayed etiological diagnosis can be associated with markedly higher morbidity and mortality. It is divided into two main types: Transudative and Exudative, depending on the mechanism of fluid accumulation. The most common causes of pleural effusion are: Congestive heart failure, infections, malignancy, liver and kidney failure. Thoracentesis may be indicated as a therapeutic procedure to relieve symptoms or as a diagnostic procedure by analyzing the pleural fluid using Light's criteria which differentiate Transudative from Exudative effusions. The main objective of this study is to determine the prevalence and etiologies of different types of pleural effusion, and to assess its relation with some potential risk factors.
\end{abstract}

Methods: This is a one-year retrospective cross-sectional analytical study. We collected data from 131 patients who underwent thoracentesis at the Lebanese Hospital Geitawi, Rafic Hariri University Hospital, and Makassed General Hospital, between January $1^{\text {st }} 2016$ and January $1^{\text {st }} 2017$.

Results: This study showed that $78 \%$ of our patients had exudative pleural effusion, while the rest $22 \%$ had transudative effusion. Cardiovascular diseases were the most common causes of transudates $(76.6 \%)$ and infectious parapneumonic effusions were the most common causes of exudates $(48.8 \%)$.

Conclusion: In Lebanon, the results were similar to those of developing countries, where the parapneumonic pleural effusion is the main cause of pleural effusion.

\section{Keywords}

Pleural effusion, Transudative, Exudative and Light's criteria

\begin{abstract}
Abbreviations
ATB: Antibioticts Treatment; CKD: Chronic Kidney Disease; CT SCAN: Computed Tomography Scan; F PAH: Familial Pulmonary Arterial Hypertension; MRI: Magnetic Resonance Imaging; TB: Tuberculosis; PMH: Past Medical History; IPAH: Idiopathic Pulmonary Arterial Hypertension
\end{abstract}

\section{Introduction}

\section{General overview}

Pleural effusion is a common problem in patients seen in internal medicine and pneumology departments [1]. It's the most common manifestation of pleural disorders [2], which reflects the failure of the mechanism controlling pleural fluid: Where the increase in filtration rate overwhelms fluid drainage [3].

Pleural fluid is similar in composition to plasma but lower in protein $(<1.5 \mathrm{~g} / \mathrm{dl}=<1 \mathrm{~g} / \mathrm{L})$. It enters the pleural space from systemic capillaries in the parietal pleurae and exits via parietal pleural stomas and lymphatics [4]. Pleural effusion results from increased fluid formation and/or decreased fluid resorption [5].

There are more than 50 recognized causes leading for pleural effusion, including diseases local to the pleura or underlying lung, systemic conditions, organ dysfunction and drugs [5].

Knowledge of the most common etiologies of pleural effusion will enable physicians to correctly choose and interpret different diagnostic tests, and to reduce mortality and morbidity rates resulting from PE complications. 
Complicated bacterial pneumonia is the leading cause of pleural effusion in developing countries, and more than $40 \%$ of pneumonia infected patients have an associated pleural effusion [2]. Any effusion in the pleura secondary to pneumonia or lung abscess will be known as parapneumonic pleural effusion [6], and should be treated with early and adequate antibiotics treatment [7].

On another hand, the most common cause of pleural effusion in USA is congestive heart failure, followed by pleural infection, and malignancy [8].

According to "world economic situation and prospects 2012", Lebanon is classified as a developing country, but there is a lack for published studies concerning pleural effusion in Lebanon.

The last Lebanese study concerning pleural effusion causes and management was done in north Lebanon in 2001 , on 165 patients with exudative pleural fluid. It showed that the main etiology of exudative effusions is tuberculosis $(43.7 \%)$, followed by malignancy $(32.1 \%)$ [9]. Since transudative effusions were not involved in this study, and data was collected from one hospital, more studies are required.

This study is also inconsistent with the report of "The joint review of the national tuberculosis programme of Lebanon" carried out by the World Health Organization, which says that "Lebanon has low TB burden".

The aim of our study was to describe the frequency, the most common etiologies, and the potential risk factors for each type of pleural effusion.

It was performed on patients admitted with pleural effusion, who underwent thoracentesis in three main university hospitals in Beirut: Lebanese Hospital Geitaoui, Rafik Hariri University Hospital and Makassed General Hospital, over one year.

\section{Literature review}

Etiology of exudative pleural effusions in adults in North Lebanon: Part of literature concerning pleural effusion prevalence, a prospective study selectively was done on exudative pleural effusion conducted of the medical records and chest radiographs of consecutive cases of exudative pleural effusions referred to the internal medicine service of the Husseini Hospital, Tripoli, Lebanon, during a three-year period from January 1997 to December 1999. 165 patients between 21 and 80 years of age with exudative pleural effusions during the study period, 114 (69.1\%) were men and 51 (30.9\%) were women. Female patients were significantly older $(P=0.05)$, and the male to female ratio was 2.3:1 with more predilection of effusion at the right side. Otherwise the study insisted that the two most common causes of exudative pleural effusions were tuberculosis (43.7\%) and malignancy (32.1\%) [9].
Patients with tuberculous pleural effusions were significantly younger than the rest $(P<0.05)$. Tuberculous effusions were more frequent in the first five decades of life ( 48 of $72=66.7 \%$ cases) and were the most common type of pleural effusion, accounting for 48 of 70 (68.6\%) patients younger than 50 years of age.

On the other hand, malignant effusions were more frequent among the older age groups-73.6\% patients with malignant effusions were older than 50 years of age. The majority of patients with empyema (81.8\%) and parapneumonic effusion (77.8\%) were older than 50 years of age.

Concerning cultures of the empyema fluid studied, the most common bacteria noted was Klebsiella pneumonia (50\% of identified bacteria in positive cultures). In contrast Klebsiella pneumonia was noted in $75 \%$ of positive parapneumonic pleural fluid culture.

Then the study concluded that tuberculosis followed by malignancy, particularly lung cancer, are the most frequent causes of exudative pleural effusion.

Etiology of pleural effusion among adults in the State of Qatar: A study on 200 patients $(73.5 \%$ were non-Qatari (mainly from Asian subcontinent) and $26.5 \%$ were Qataris residents) with pleural effusion done in Hamad General Hospital- state of Qatar, over 1 year in 2009 published on "Eastern Mediterranean health journal", showed that tuberculosis represents the most common cause of pleural effusion (32.5\%), followed by parapneumonic effusion, malignancy and heart failure [10].

According to light criteria, $79 \%$ of PE were exudates, and $21 \%$ were transudate; among exudates, TB was the most common cause (41\%), followed by parapneumonic effusions (24\%), malignancy (mainly lung cancer) (19.6\%), and empyema (10.8\%). Among transudates, heart failure was the most frequent etiology in elderly patients (mainly due to ischemic heart diseases) with a percentage of $62 \%$, followed by liver failure $16.7 \%$ and kidney disease $14.3 \%$.

The study also noted difference between locations of effusions, $54.5 \%$ were located at the right side (mostly caused by TB), $31.5 \%$ located at the left side, and $14 \%$ were bilateral (mainly from cardiac origin). In the parapneumonic pleural effusions, blood cultures were positive in $23.7 \%$, where strep pneumonia (66.7\%) was the most common germ, followed by Klebsiella pneumonia (22.2\%) and Staph aureus (11\%). Cultures of pleural fluid from patients with empyema were positive in $47 \%$ of cases, gram-positive organisms accounted for $62.5 \%$ of all isolates, and Streptococcus milleri was the most encountered aerobic Gram-positive organism.

Pleural effusions from congestive heart failure: $A$ more specific literature review concerning heart failure induced transudative pleural effusion in Spain by Jose 
M. Porcel, where data collected from the pleural disease unit, department of internal medicine in Arnau de Vilanova university hospital published in 2010: Among 2388 patients who underwent diagnostic thoracentesis for pleural effusion during the past 15 years, main causes were cancer (28.5\%); pneumonia (19.5\%); heart failure (18\%) and tuberculosis (9\%) [11].

The study additionally added that $78.5 \%$ of transudate was secondary to heart failure, and $13 \%$ to liver failure; it also showed that light criteria correctly classified $97.5 \%$ of exudates and $74 \%$ of transudates, making it the gold standard method for recognizing the type of pleural effusion.

Mortality of Hospitalized patients with pleural effusions: Concerning mortality of patients with pleural effusion, a retrospective chart reviews all patients admitted to Yale-New Haven Hospital medical floors during March 2011. Of all patients who had chest radiograph on admission, pleural effusion was identified in $14 \%$ of them. Severity of illness, and a diagnosis of cancer increase the mortality at both 30-days and 12-months. The study also demonstrated: $35 \%$ 30-day mortality in patients with MPE, $26 \%$ mortality in patients with multiple benign etiologies, and $7-14 \%$ mortality in patients with transudative causes [12].

The article demonstrated that mortality within 30 days of admission occurred in $15 \%$ of patients with pleural effusion, and that the mortality within one year of admission, increased to $36 \%$ in patients who needed thoracentesis.

Finally, the article concluded the following: "Pleural effusions may serve as a marker of mortality in patients admitted to general medical services".

Study of 100 cases of pleural effusion with reference to diagnostic approach: A study done in 2016 in the Department of Pulmonary Medicine, B.J. Medical College, Ahmedabad, India, showed that among 100 indoor adult patients with clinical and radiographic diagnosis of pleural effusion, and underwent thoracentesis, $91 \%$ have exudative pleural effusion divided into: Tuberculous effusion (62\%), malignant pleural effusion (18\%) and parapneumonic effusion (10\%). Among 62 patients of tuberculous effusion only 4 patients had past history of tuberculosis, and among 18 patients with malignant pleural effusion, only 9 patients have positive pleural fluid cytology confirmed by biopsy. The majority of these patients were young, between 31 and 50 years, and pleural effusion was more common in males $(68 \%)$ than females (32\%). Unilateral effusion, mainly at the right side $(60 \%)$, was the most common location of effusion [13].

This study showed also that thoracentesis followed by pleural fluid analysis is the best method to diagnose the underlying etiology, and undiagnosed pleural effusions can be best diagnosed by thoracoscopic pleural biopsy and histopathological analysis, which gave $100 \%$ diagnosis.

Exudative pleural effusion: Effectiveness of pleural fluid analysis and pleural biopsy: In Iran, a study on 100 patients with Pleural Effusion admitted between 1997 and 2001 showed that the mean age of patients was 57 years, and the majority of patients were males (62\%) [14].

The study demonstrates that the most common cause of pleural effusion was Malignancy (41\%), followed by Tuberculosis (33\%), other causes were: Parapneumonic effusions (6\%), congestive heart failure (3\%), complications of coronary bypass surgery (2\%), Rheumatoid arthritis (2\%), SLE (1\%), CRF (1\%), acute cholecystitis (1\%), unknown etiologies (8\%).

It also mentioned that the majority of malignant pleural effusions were due to metastatic cancer (95\%), but the origins of primary cancer were only determined in only $(39 \%)$ of patients, which included lung cancer $(22 \%)$, breast cancer $(7 \%)$, gastric carcinoma $(5 \%)$, lymphoma (5\%), and malignant mesothelioma (2\%).

Pleural effusion in adults-etiology, diagnosis, and treatment: According to an article published by the Department of Respiratory Medicine, Hannover Medical School, it can be estimated, that 400,000 to 500,000 persons per year in Germany suffer from pleural effusion.

In this study they found out that the most common causes of pleural effusion are congestive heart failure, cancer, pneumonia, and pulmonary embolism.

A number of rarer diseases can be associated with pleural effusion, almost always of the exudative type. $30-50 \%$ of patients with systemic lupus erythematosus have a pleural effusion ("polyserositis"). Pleural effusion is also not infrequently seen in patients suffering from granulomatosis with polyangiitis (Wegener disease), rheumatoid arthritis, and Langerhans-cell granulomatosis.

$21 \%$ of patients suffering from idiopathic and familial pulmonary hypertension (iPAH and $\mathrm{PPAH}$ ) have a pleural effusion, mostly unilateral.

One of the more common causes of unexplained pleural effusion is pulmonary embolism. $20-55 \%$ of patients with pulmonary embolism have a pleural effusion. Bintcliffe, et al. found that $70 \%$ of 126 patients with a pleural effusion did, indeed, have a single cause for it, but $30 \%$ had more than one cause [15].

\section{Objectives}

As we discussed above, pleural effusion is comorbid condition that increases mortality and needs specific work-up and close follow-up, with a wide differential diagnosis where cooperation between several medical specialties should occur. Otherwise no recent studies, 
articles, nor data concerning pleural effusion in Lebanon were published in the last 18 years.

In absence of recent studies and data in Lebanon, our study on pleural effusion etiologies in three major hospitals in Beirut: Lebanese Hospital Geitawi, Rafic Hariri University Hospital, and Makassed General Hospital, could help the physicians to treat the underlying disorders, avoid invasive diagnostic tests when not needed, decrease mortality from pleural effusion, and maybe to find ways to prevent the disease in many patients.

\section{Primary objective:}

- To evaluate the frequency of each type of pleural effusion admitted to these university hospitals between transudative versus exudative depending on light criteria.

\section{Secondary objectives:}

- To determine the total number of admissions with pleural effusion who underwent a thoracentesis over 1 year in three university hospitals in Beirut.

- To identify the most common etiology for each type of pleural effusion in our study population.

- To assess the presence of potential risk factors for each type of pleural effusion: age, gender, and comorbidities.

Hypothesis: We assume that Lebanon is classified as a developing country, and that the number of exudative effusions is superior to that of transudative effusions.

\section{Subjects and Methods}

\section{Study design}

This is a one-year retrospective cross-sectional analytical study. We collected data from 131 patients who underwent thoracentesis at the Lebanese Hospital Geitawi, Rafic Hariri University Hospital, and Makassed General Hospital between January $1^{\text {st }} 2016$ and January $1^{\text {st }}$ 2017.

\section{Study population}

Population studied: The study population consisted of patients labeled as having the diagnosis of pleural effusion on admission or upon discharge between January $1^{\text {st }} 2016$ and January $1^{\text {st }} 2017$. The sample was collected from those admitted in three university hospitals in Beirut: Lebanese Hospital Geitaoui, Rafik Hariri University Hospital and Makassed General Hospital. It consisted of 131 patients.

No written consent was necessary from the patients included in our study, nor from their physicians, because it was leaded using the medical record from the hospitals archives. A letter signed from the Lebanese University was presented to the ethical committee of the hospitals, in order to access the patient's medical records from their archive.

\section{Inclusion criteria:}

- Patients aged more than 16 years.

- Having official diagnosis of pleural effusion based on radiological means and thoracentesis findings.

Exclusion criteria:

- Patients aged less than 16 years.

- Having non-investigated pleural effusion diagnosis (no thoracentesis findings files).

\section{Procedures and Statistical Analysis}

\section{Data collection procedure}

Data was collected from patients labeled as having the diagnosis of pleural effusion on admission or upon discharge between January $1^{\text {st }} 2016$ and January $1^{\text {st }} 2017$ in three university hospitals in Beirut: Lebanese Hospital Geitaoui, Rafik Hariri University Hospital and Makassed General Hospital. Data was collected by three students from the Faculty of Medicine of the Lebanese University, using a modified standardized checklist containing the light's criteria used worldwide and the main factors involved in the field of our study [16-18].

\section{Variables}

The datasheet we filled had several interest variables:

- Age

- Gender

- Past medical history

- Imaging (X-ray, CT scan, Ultrasound)

- Pleural fluid analysis by thoracentesis (cytology, biochemistry, microbiology)

- Blood samples for levels of total proteins, albumin, lactate dehydrogenase, glucose

- Official diagnosis by treating physician

- Treatment used

- Patient discharge status

\section{Statistical analysis}

We used Excel for data entry and for the tables and graphs used as study results. We used SPSS version 23 for data analysis. A descriptive analysis was first done to assess the distribution of all variables. Then, potential associations between two categorical variables were assessed using Pearson's Chi square Test. Anova relations were studied between continuous variables and the contributor. The significance level of the test was admitted as $p$-value $<0.05$.

\section{Results}

\section{Univariate analysis}

Age distribution (\%): Figure 1 shows that the major- 


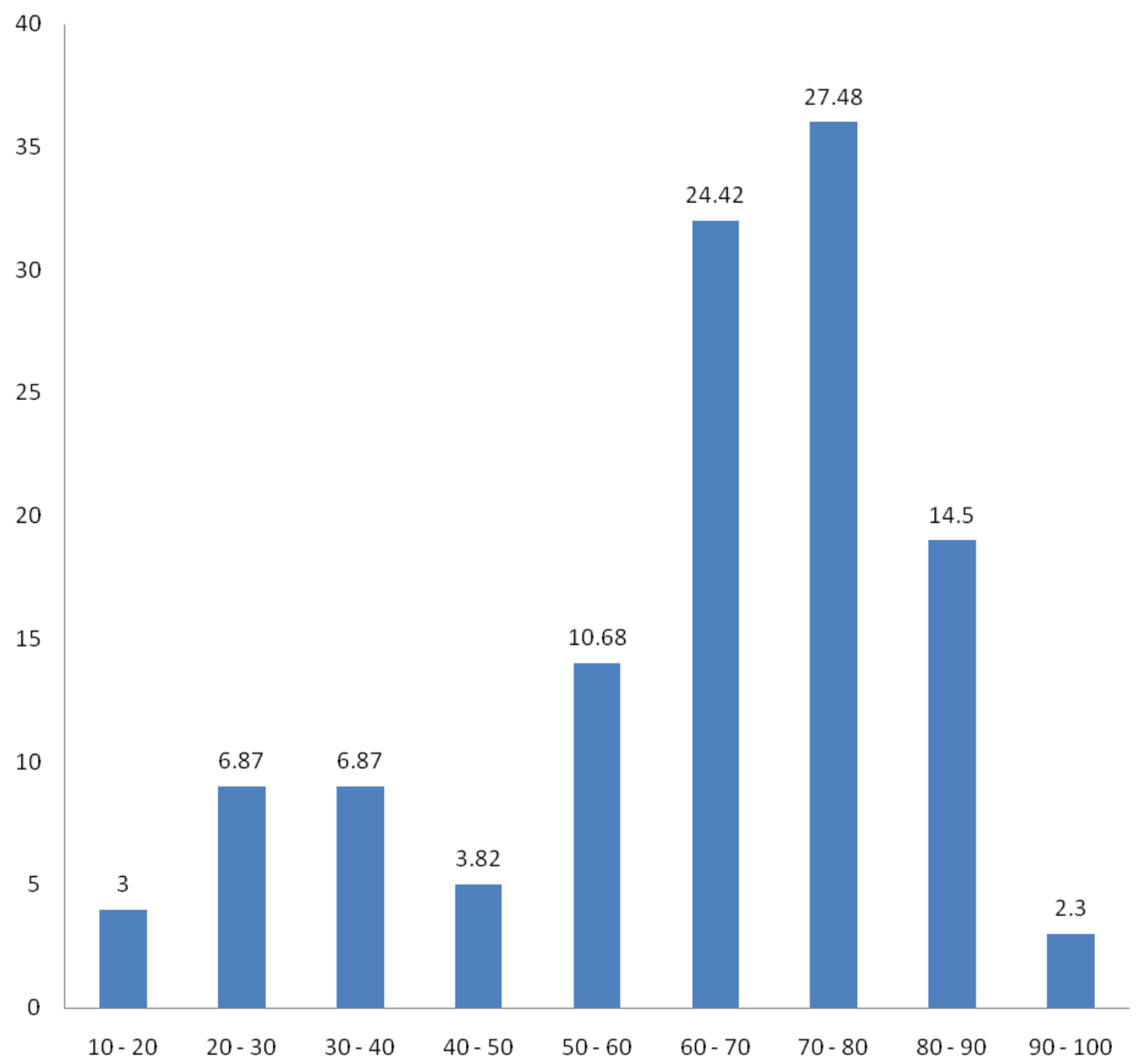

Figure 1: Shows that the majority of our sample $(90 / 131=68.7 \%)$ were seniors aged more than 60 -years-old.

Table 1: As for the previous medical history, the majority (55\%) of the patients had cardiovascular diseases, while only $1.5 \%$ had infectious empyema.

\begin{tabular}{|l|l|l|}
\hline Factors & Frequency & Percent \\
\hline Cardiovascular & 72 & 55 \\
\hline Other PMH & 48 & 36.6 \\
\hline None & 28 & 21.4 \\
\hline CKD & 17 & 13.0 \\
\hline Metastasis & 13 & 9.9 \\
\hline Primary malignancy & 8 & 6.1 \\
\hline Neurological disease & 5 & 3.8 \\
\hline Infectious empyema & 2 & 1.5 \\
\hline Infectious parapneumonic & 1 & 0.8 \\
\hline Inflammatory autoimmune & 0 & 0.0 \\
\hline Liver failure & 0 & 0.0 \\
\hline
\end{tabular}

ity of our sample $(90 / 131=68.7 \%)$ were seniors aged more than 60-years-old.

Gender distribution: Half of our sample (52\%) consisted of men as shown in Figure 2.
Past medical history: Figure 3 shows that half of our sample (51\%) had more than one disease in their past medical history, while $27 \%$ didn't have any previous diseases.

As for the previous medical history, the majority (55\%) of the patients had cardiovascular diseases, while only $1.5 \%$ had infectious empyema (Table 1 ).

Imaging diagnosis source (\%): It's noted that almost half of the patients $48.85 \%$ (64/131) underwent several imaging procedures in order to have their diagnosis defined (X-Ray \& CT scan). Thus, only few patients $0.76 \%(1 / 131)$ had an extra MRI. However, almost $45 \%$ $(59 / 131)$ of the patients were diagnosed just after an $\mathrm{X}$-Ray alone (Figure 4).

Imaging results: Two thirds (66\%) of imaging results were unilateral, as showed by the Figure 5 .

Diagnosis using light criteria: Figure 6 shows that the vast majority of our patients $(78 \%)$ had exudate effusions according to Light's criteria, while the rest (22\%) had transudate effusions. 

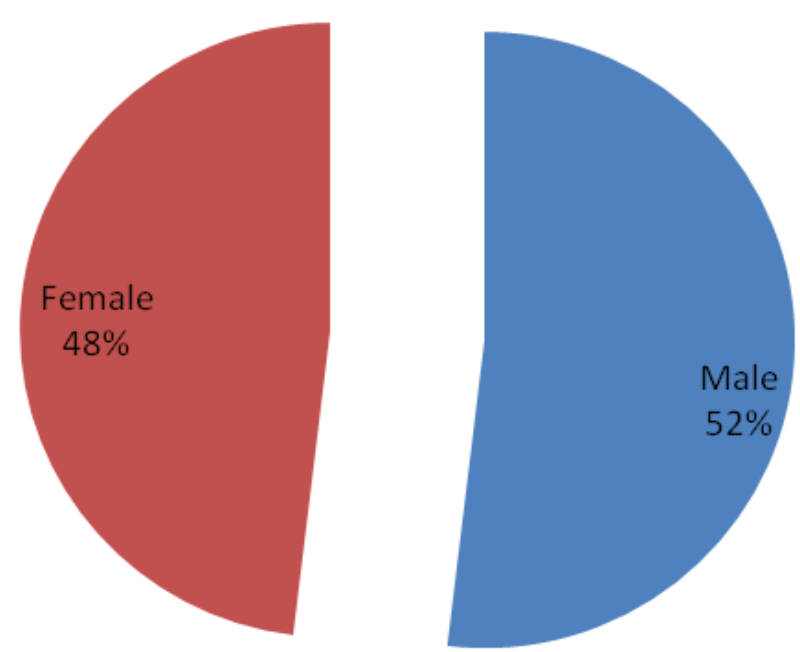

Figure 2: Half of our sample $(52 \%)$ consisted of men as shown above.

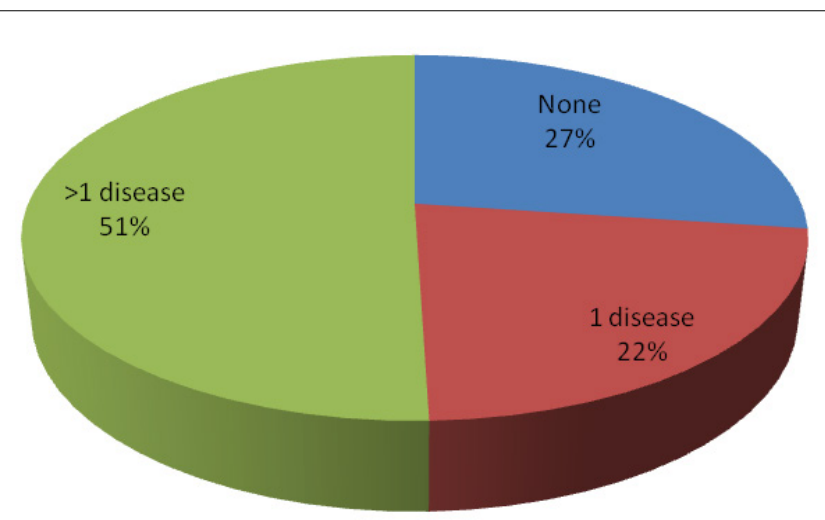

Figure 3: This Figure shows that half of our sample (51\%) had more than one disease in their past medical history, while $27 \%$ didn't have any previous diseases.

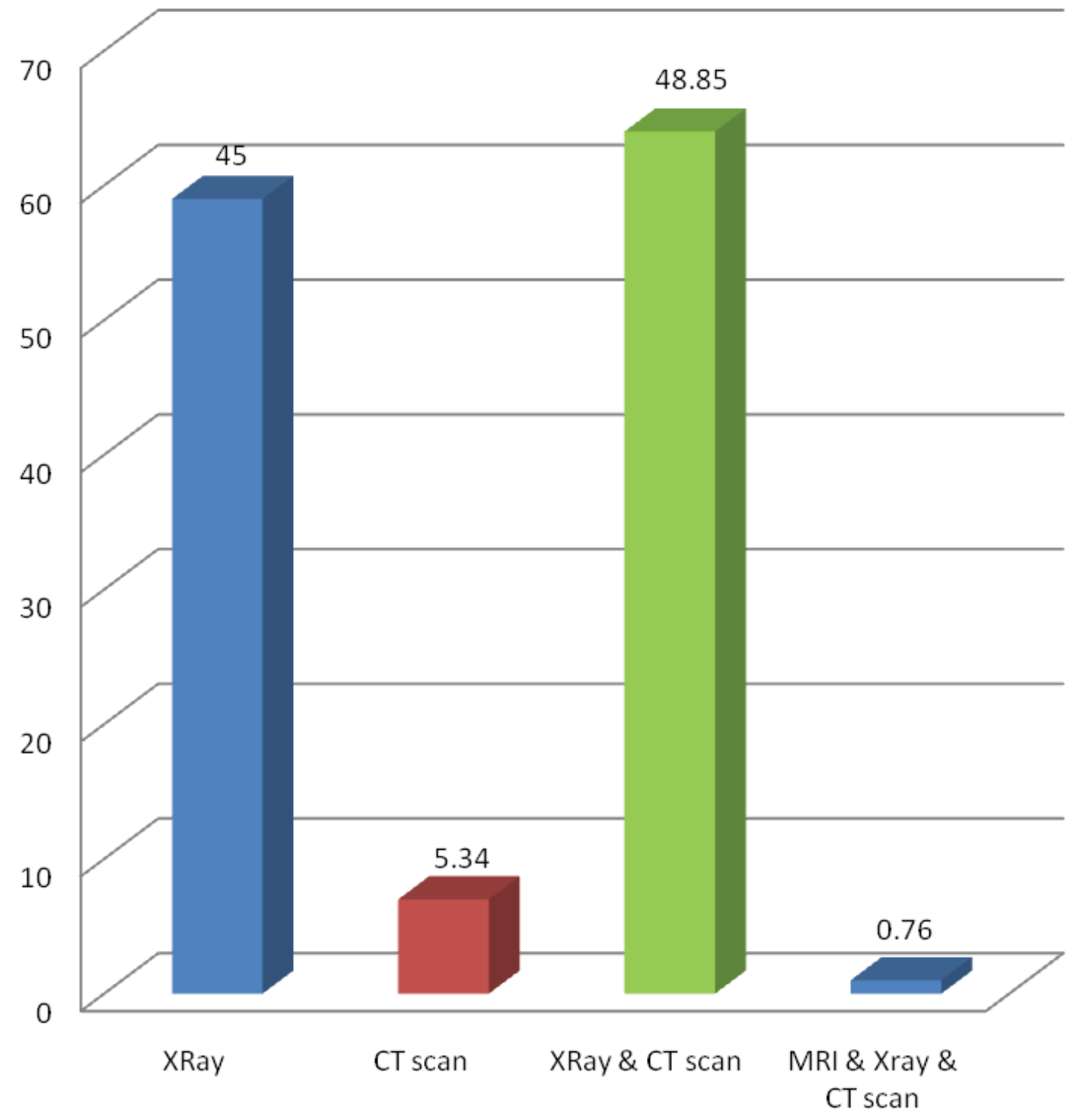

Figure 4: It's noted that almost half of the patients $48.85 \%$ (64/131) underwent several imaging procedures in order to have their diagnosis defined (X-Ray \& CT scan). Thus, only few patients $0.76 \%(1 / 131)$ had an extra MRI. However, almost $45 \%$ $(59 / 131)$ of the patients were diagnosed just after an X-Ray alone. 


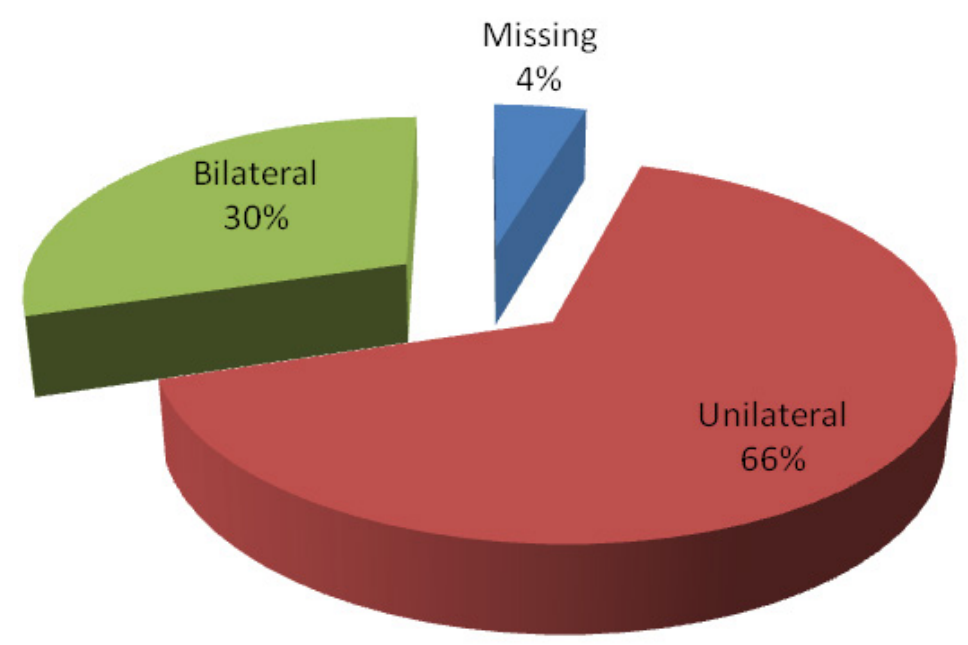

Figure 5: Two thirds (66\%) of imaging results were unilateral, as showed by the figure above.

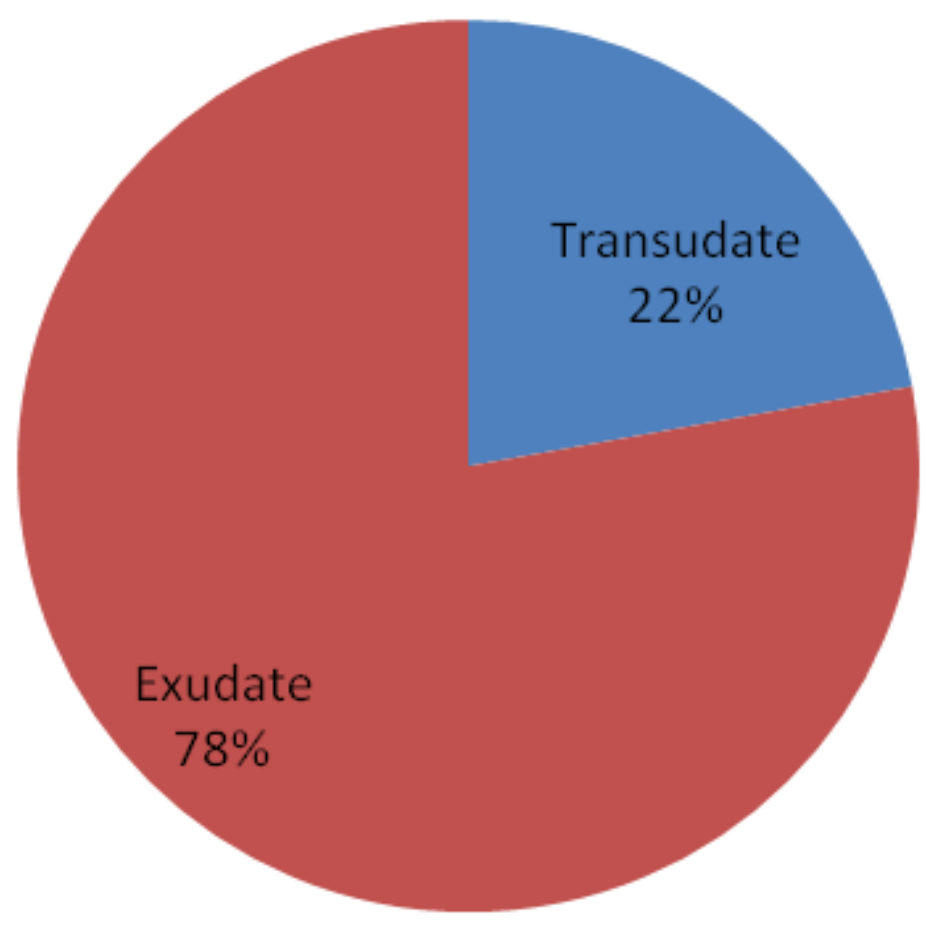

Figure 6: This figure shows that the vast majority of our patients $(78 \%)$ had exudate effusions according to Light's criteria, while the rest $(22 \%)$ had transudate effusions.

Reported transudative or exudative effusions: The majority of the transudative lesions $(23 / 30=76.67 \%)$ were from cardiovascular origin. Infectious parapneumonic type was the most common among exudative lesions $(41 / 84=48.8 \%)$, while inflammatory ones were the least frequent. However, we have 17 cases missing according to files findings (Figure 7).

Microbiology results: The majority of our patients $(99 / 131=75.6 \%)$ had negative microbiology results, while a minority of cases $(9 / 131=7 \%)$ reported an identification of an infectious agent among exudate pleural effusion whereas Tuberculosis represent the most common germ (Figure 8).
Treatment used for transudative effusions: Figure 9 shows the treatment options that the patients received when they were diagnosed as having transudate effusion. The majority of them $(11 / 29=38 \%)$ were treated with diuretics and antibiotic together, while only 1 patient was treated with antibiotics and chest tube.

Treatment used for exudative effusions: Figure 10 shows that the majority of the patients, who have exudate effusions, were treated by antibiotics and chest tube or by antibiotics alone (respectively $28 / 103=27 \%$ and $23 / 103=22 \%$ ).

Discharge status: The reported discharge status of the majority of the patients (65\%) does not show any 


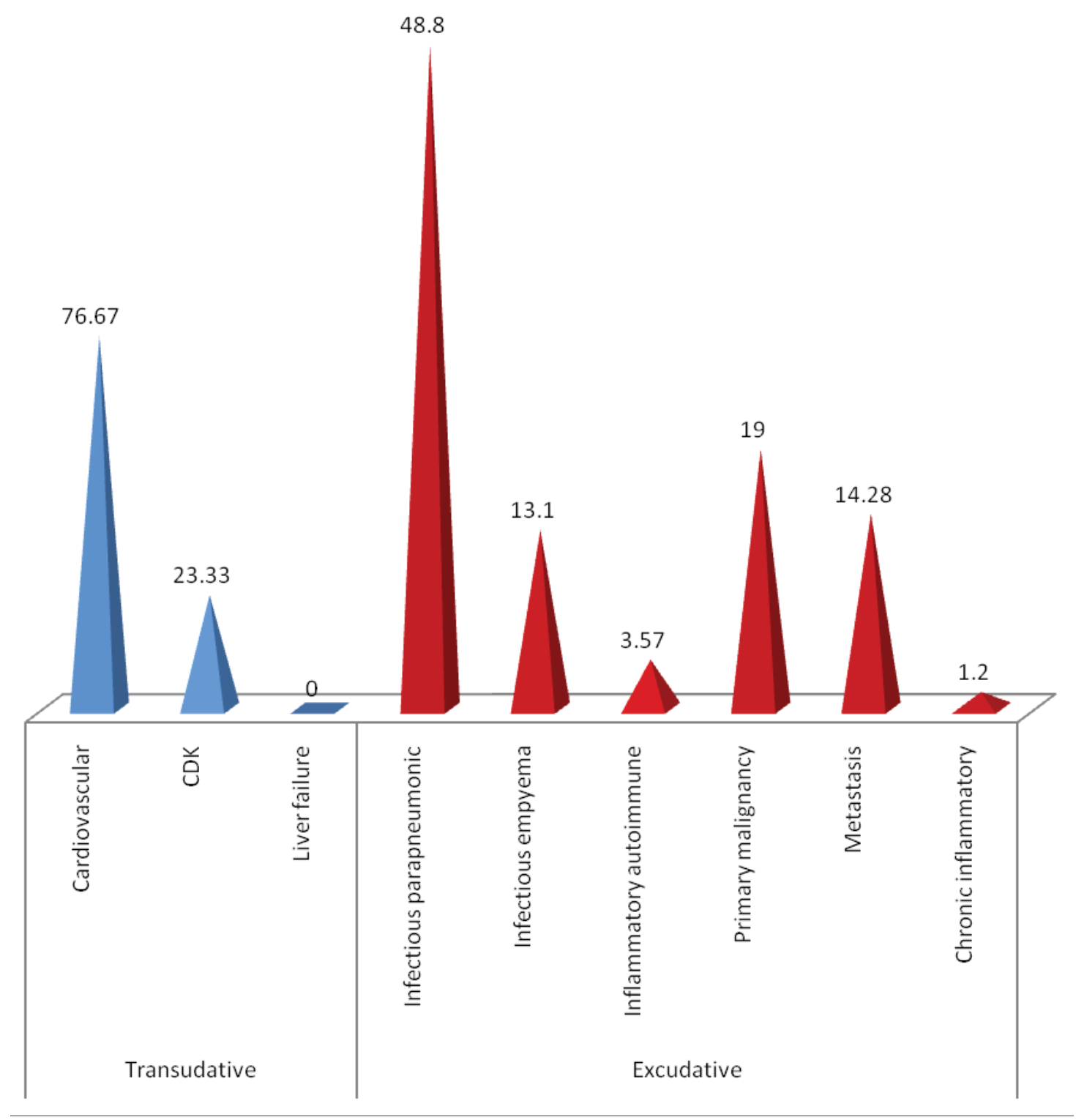

Figure 7: The majority of the transudative lesions $(23 / 30=76.67 \%)$ were from cardiovascular origin. Infectious parapneumonic type was the most common among exudative lesions $(41 / 84=48.8 \%)$, while inflammatory ones were the least frequent. However, we have 17 cases missing according to files findings.

complications during hospitalization. However, $15 \%$ had complications, and 16\% were dead (Figure 11) (Annex).

\section{Bivariate analysis}

Diagnosis using light's criteria cross tabulation across age categories: Discussing the relation between age and type of effusion presented, it was found that those in between the age categories $61-80$ are of highest frequencies in both exudate and transudate types (39 and 22 patients respectively). As for the transudate type, no younger age groups were involved in the diagnosis whereas to those in exudative type of effusion there are 31 patients who are less than 60-years-old. A significant $p$-value of 0.018 reveals that transudative type of effusion is related to age, and as age increases the possibility of presenting with this type increases. As for exudates, $p$-value of 0.267 is not significant between age and type of effusion (Table 2).
Diagnosis using light's Criteria Cross tabulation across gender: There is not enough statistical evidence to claim an association between the gender groups in terms of diagnosis using Light's criteria ( $p$-value $=0.408$ $>0.05$ ) (Table 3).

Discharge status Cross tabulation across age categories: The larger frequencies to those who had complications or morbidities from pleural effusion were 13 patients of age greater than 60 whereas only 7 patients had complications of age below 60. $P$ value $=0.047$ was obtained, showing that as age increase the morbidity rates increase. 16 patients died after developing pleural effusion above 60 -years-old. On the other hand, only 5 patients below 60 years died out of the total 21. A significant $p$-value $=0.03$ was obtained showing that as age increases the probability of mortality outcomes increases (Table 4). 


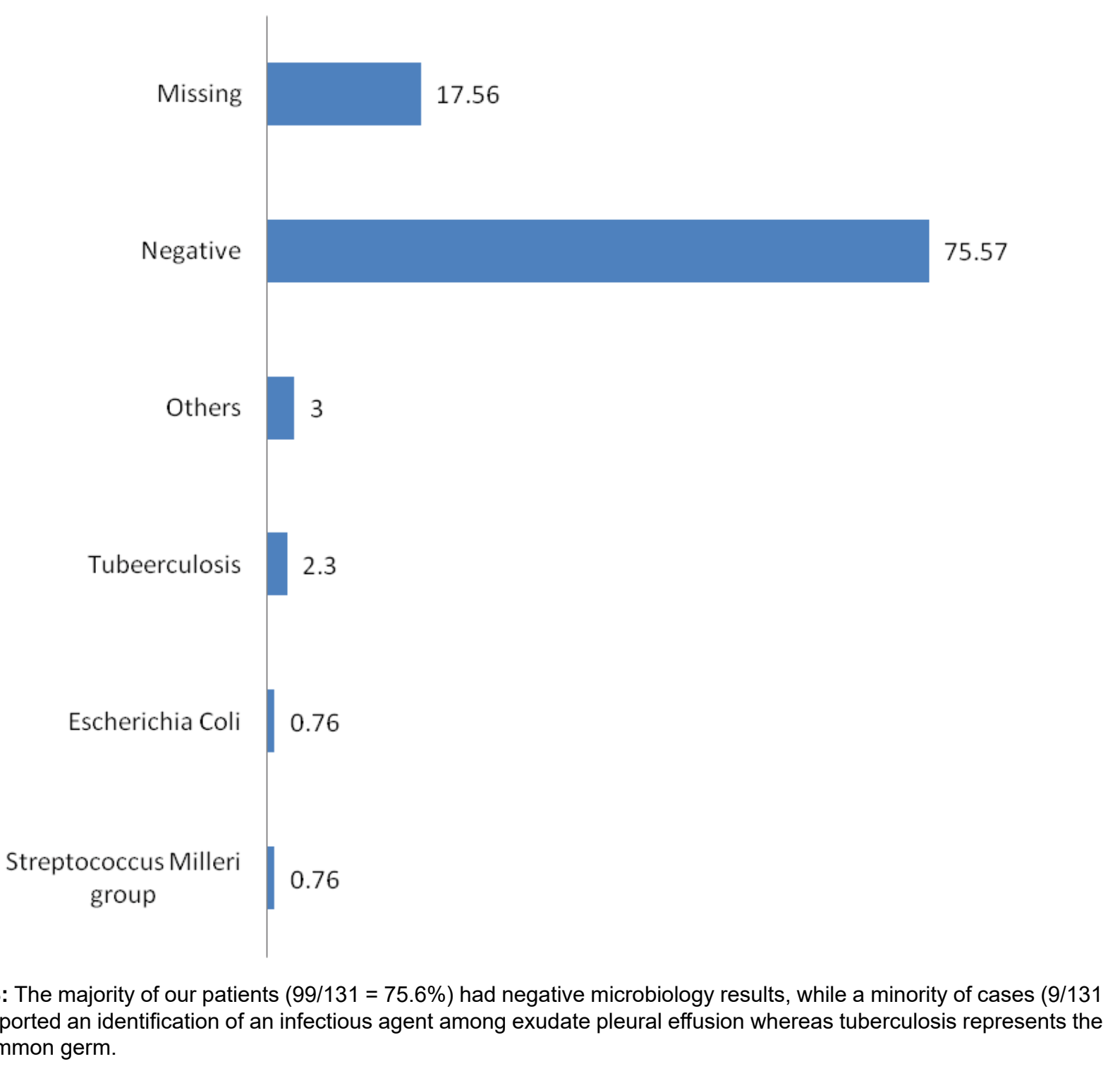

Figure 8: The majority of our patients $(99 / 131=75.6 \%)$ had negative microbiology results, while a minority of cases $(9 / 131$ $=7 \%$ ) reported an identification of an infectious agent among exudate pleural effusion whereas tuberculosis represents the most common germ.

Table 2: Diagnosis using light's criteria cross tabulation across age categories.

\begin{tabular}{|l|l|l|l|l|l|l|l|}
\hline & \multicolumn{4}{l}{ Age categories } & & Total & P-value \\
\cline { 2 - 7 } & $\mathbf{2 0}$ & $\mathbf{2 0 - 4 0}$ & $\mathbf{4 1 - 6 0}$ & $\mathbf{6 1 - 8 0}$ & $\mathbf{8 1 - 1 0 0}$ & & \\
\hline Transudate & 0 & 0 & 2 & 22 & 6 & 30 & 0.018 \\
\hline Exudate & 4 & 14 & 13 & 39 & 14 & 84 & 0.267 \\
\hline
\end{tabular}

Table 3: Diagnosis using light's criteria cross tabulation across gender.

\begin{tabular}{|l|l|l|l|}
\hline Diagnosis using light's criteria & \% within gender & Total \\
\cline { 2 - 3 } & Men & Women & \\
\hline Transudate & $19.10 \%$ & $25.40 \%$ & $22.10 \%$ \\
\hline Exudate & $80.90 \%$ & $74.60 \%$ & $77.90 \%$ \\
\hline p-value & & 0.408 & \\
\hline
\end{tabular}

Table 4: Discharge status Cross tabulation across age categories.

\begin{tabular}{|l|l|l|l|l|l|l|l|}
\hline \multirow{2}{*}{$\begin{array}{l}\text { Discharge } \\
\text { status }\end{array}$} & \multicolumn{9}{|c|}{ Age categories } & Total & P-value \\
\cline { 2 - 8 } & $\mathbf{2 0}$ & $\mathbf{2 0 - 4 0}$ & $\mathbf{4 1 - 6 0}$ & $\mathbf{6 1 - 8 0}$ & $\mathbf{8 1 - 1 0 0}$ & & \\
\hline Morbidity & 0 & 4 & 3 & 9 & 4 & 20 & 0.047 \\
\hline Mortality & 0 & 0 & 5 & 10 & 6 & 21 & 0.03 \\
\hline
\end{tabular}




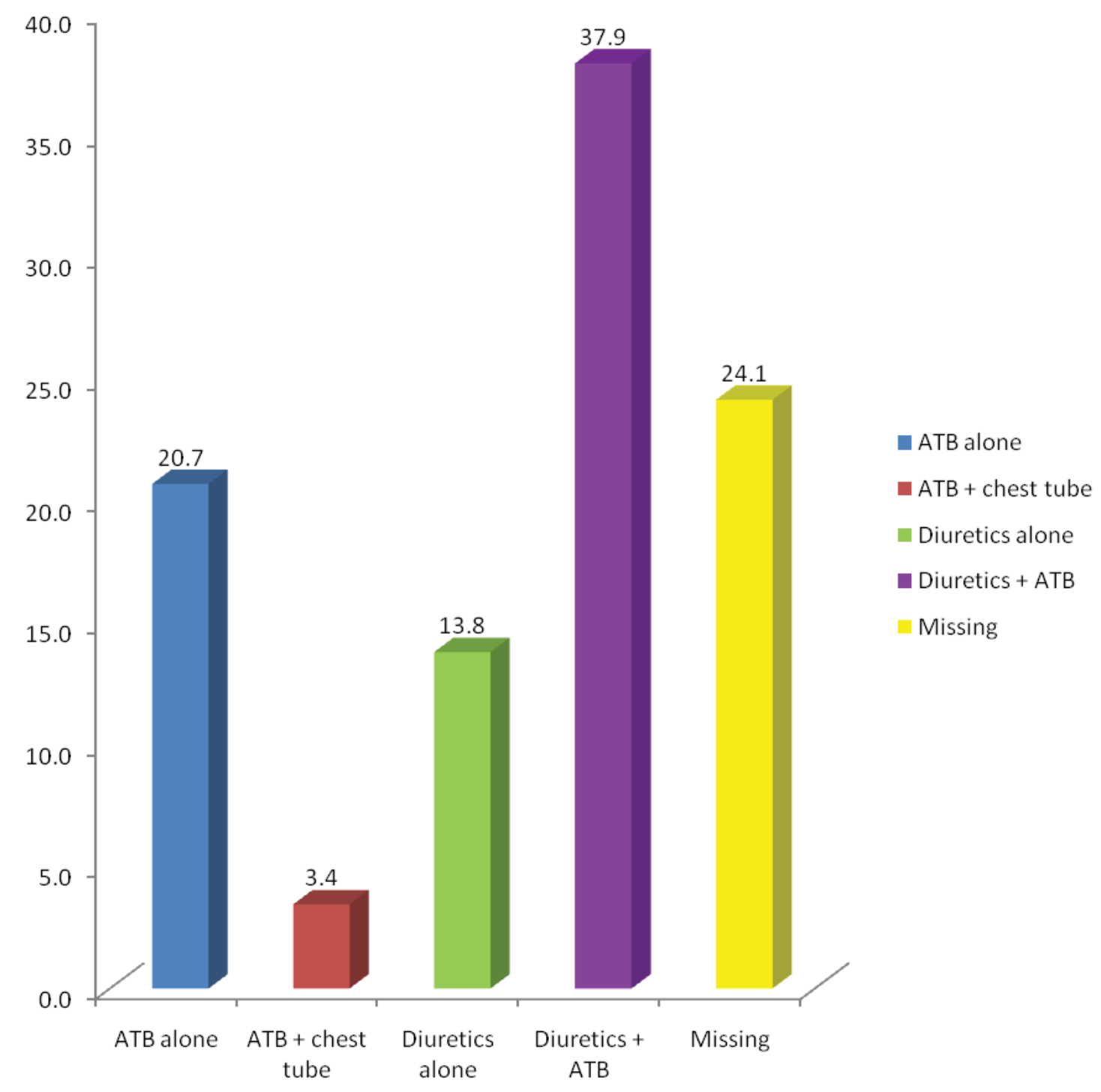

Figure 9: This figure shows the treatment options that the patients received when they were diagnosed as having transudate effusion. The majority of them $(11 / 29=38 \%)$ were treated with diuretics and antibiotic together, while only 1 patient was treated with antibiotics and chest tube.

Table 5: Discharge status cross tabulation according to gender.

\begin{tabular}{|l|l|l|l|l|}
\hline Discharge status & Men & Women & Total & P-value \\
\hline Morbidity & 6 & 14 & 20 & 0.079 \\
\hline Mortality & 9 & 12 & 21 & 0.328 \\
\hline
\end{tabular}

Table 6: Discharge status cross tabulation according to light's criteria diagnosis.

\begin{tabular}{|c|c|c|c|c|c|c|c|c|}
\hline \multirow{3}{*}{$\begin{array}{l}\text { Discharge } \\
\text { status }\end{array}$} & \multicolumn{6}{|c|}{ Type of pleural effusion } & \multirow[t]{3}{*}{ Total } & \multirow[t]{3}{*}{ P-value } \\
\hline & \multicolumn{2}{|c|}{ Transudate } & \multicolumn{2}{|c|}{ Exudate } & \multicolumn{2}{|c|}{ Missing } & & \\
\hline & Count & $\%$ & Count & $\%$ & Count & $\%$ & & \\
\hline Morbidity & 4 & 20 & 11 & 55 & 5 & 25 & 20 & 0.03 \\
\hline Mortality & 6 & 28.6 & 13 & 61.9 & 2 & 9.5 & 21 & 0.04 \\
\hline
\end{tabular}

Discharge status Cross tabulation according to gender: In both morbidity and mortality females tend to be of higher frequencies 14 out of 20 and 12 out of 21 respectively. P-values of 0.079 and 0.0328 demonstrate that no significance between genders presented with effusion and discharge status of these patients (Table 5).

Discharge status Cross tabulation according to
Light's Criteria diagnosis: 13 out of 21 patients who were found to have an exudative effusion died afterward which is greater than those who had transudative type of effusion which were 6 . In addition, 11 out 20 patients with exudative effusion present complications. However, only 4 patients with transudative effusion present complications. Significant P-value of 0.04 and 
0.03 confirm that the exudative type of effusion causes higher rates of mortality and morbidity respectively than transudative type (Table 6).

\section{Discussion}

The main purpose of this study is to determine the prevalence and the etiologies of transudate and exudate effusions among Lebanese patients hospitalized for pleural effusion, and to correlate pleural effusion with some potential risk factors.

We have found that among our patients with pleural effusion who underwent thoracentesis, $78 \%$ of the cases meet the biochemical characteristics of exudative fluid, according to light criteria, while $22 \%$ display transudative pleural effusion (Figure 6).

The most frequent etiology of exudative lesion is infection, with a predominance of tuberculosis (3 cases among 9 positive pleural fluid cultures) presented with parapneumonic effusion (48.8\%), empyema (13\%), and followed by malignancy, including primary malignancy and metastasis ( $16 \%$ and $12 \%$ respectively). Nevertheless, the most common etiology of transudative lesions is linked to a cardiac origin (76.67\%) which is found in $55 \%$ of the patients' previous medical history (Table 1 ), followed by chronic kidney disease in $23.3 \%$ of cases (Figure 7). We can conclude that patients with cardiac diseases may be at high risk to develop pleural effusion, mainly the transudative type.

These results were similar to other studies done in developing countries, such as India [13] where $90 \%$ are exudates: Tuberculosis attacks the vast majority of patients (62\% of cases) and malignancy represents $18 \%$ of cases. In Qatar [10], 79\% of the cases reveal exudates with a predominance of tuberculosis (41\%) followed by parapneumonic effusion (24\%). Same conclusion is settled in previous studies done in North Lebanon [9], Iran [14], and Kuwait [15] where tuberculosis and malignancy are the main cause of exudate pleural effusion.

Contrarily, in developed countries: the leading cause of pleural effusion is transudative effusion. For instance, in USA, 500,000 cases/year are triggered by congestive heart failure, 300,000 cases/year are caused by pneumonia, and 200,000 cases/year are due to malignancy [16]. Furthermore, in Germany, a study based on data of 400,000 to 500000 individuals who are diagnosed with pleural effusion, revealed that congestive heart failure is on the top of the etiologies list, followed by cancer, pneumonia, and pulmonary embolism [17].

This contrast in the results may be linked to a poor socioeconomic status, lifestyle quality, and often the inadequate healthcare system in developing countries which heightens the risk to develop exudative effusion via infection and malignancy. Another hypothesis which may explain the low incidence of transudative effusion is that the majority of this group is treated with diuret- ics and fluid restriction, based on history and physical examination. Hence, they rarely need thoracentesis as diagnostic or therapeutic procedure.

As for the age and gender distribution, we observed that $68.7 \%$ of our patients are elderly (above 60 -yearsold) with slight predominance of male (52\%) over female patients (48\%) (Figure 1 and Figure 2). We deduce that age is an essential risk factor to develop pleural effusion in adults; particularly transudative type with a significant $\mathrm{P}$-value of 0.018 , due to the presence of some previous comorbidities related to this age group, but there is no role for gender in the development and classification of pleural effusion (Transudate versus Exudate) like the majority of results worldwide [1] (Table 2 and Table 3). In other studies, investigating the effect of demography on the etiology of pleural effusion, we noted that infectious pleural effusion caused by tuberculosis is more frequent in young population ( $<50$ years). The malignant effusions, and transudate effusions caused by cardiac failure were more frequent among older age groups (> 50-years-old) $[9,10]$.

Concerning the diagnostic methods, chest radio-graphs alone usually verify the presence of a pleural effusion, and computed tomography is occasionally used in case of doubt, or to detect small effusions or to differentiate pleural fluid from pleural thickening [16]. Nonetheless, most of our cases (48.85\%) needed both $\mathrm{X}$-Ray \& CT scan in order to reach a definitive diagnosis, which showed unilateral pleural effusion in two thirds $(66 \%)$ of cases (Figure 5$)$. Some studies established a relationship between the location of pleural effusion with its type: Unilateral effusion mainly on the right side is more frequent in patients with exudative infectious and malignant effusions, and bilateral effusion is shown predominantly in transudate effusions caused by heart failure $[10,13,16]$.

We observed a high incidence of negative microbiology results $(75.6 \%)$, which is much higher than other studies, where $48 \%$ of cultures from empyema identified pathogens, and $31 \%$ of cases were positive from all other origins of effusion [18]. Our findings can be associated with several factors, such as an infection with a virus or mycoplasma, or a prior empiric antibiotic therapy. To elaborate further, antibiotics are used in around $60 \%$ of patients with exudates: $22.5 \%$ of patients are treated with antibiotics alone, $27.5 \%$ in combination with chest tube, and $9.8 \%$ with diuretic. Moreover, even in transudative effusions, where diuretics are the first therapeutic option, antibiotics were also frequently used: Around $38 \%$ of cases are treated with diuretics in addition to antibiotherapy. To summarize, antibiotics are excessively prescribed in our population, which may be due to misdiagnosis, or due to other syndromes unrelated to pleural effusion requiring antiobiotherapy (Figure 8, Figure 9 and Figure 10). 


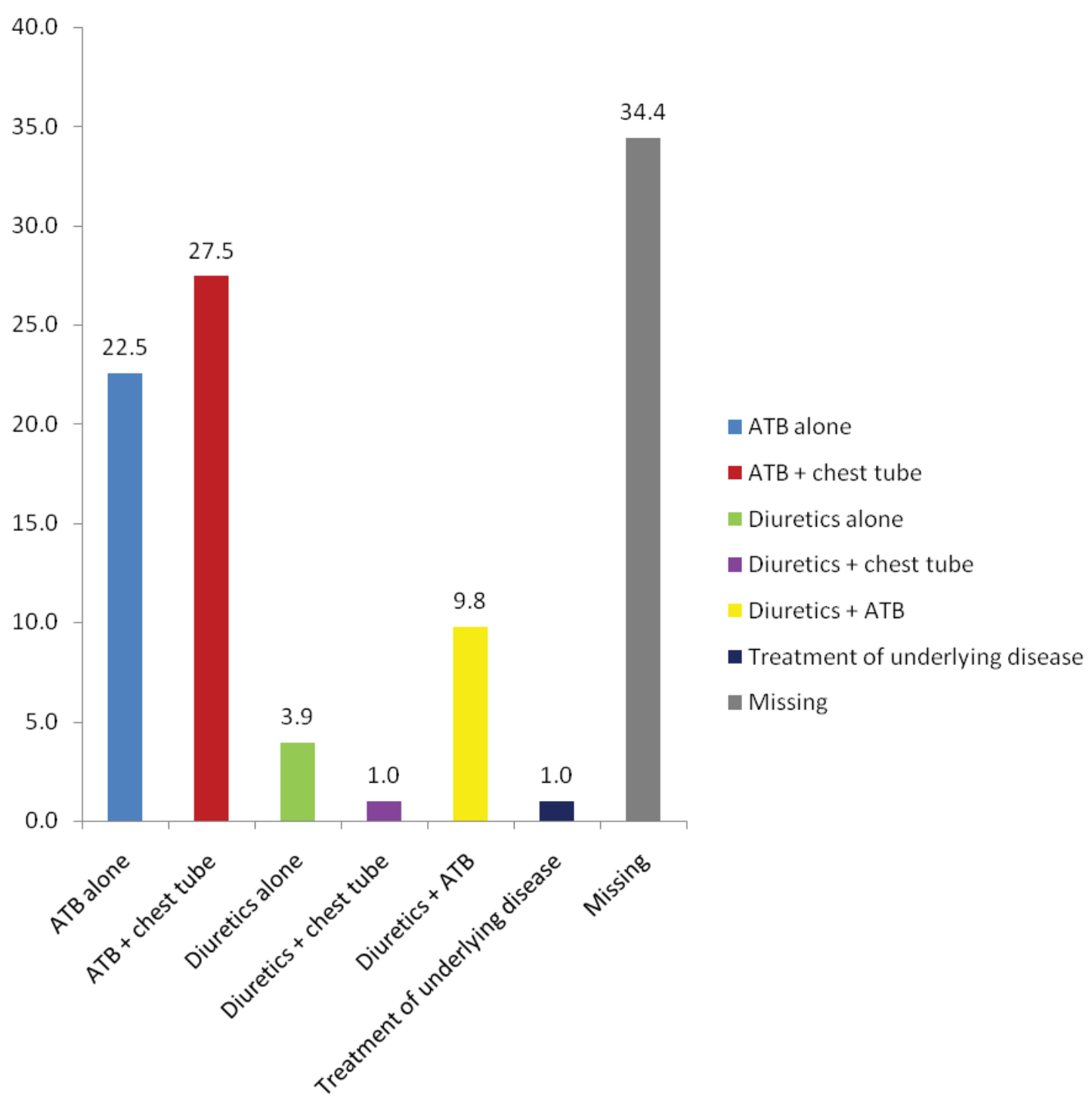

Figure 10: This Figure shows that the majority of the patients, who have exudate effusions, were treated by antibiotics and chest tube or by antibiotics alone (respectively $28 / 103=27 \%$ and $23 / 103=22 \%$ ).

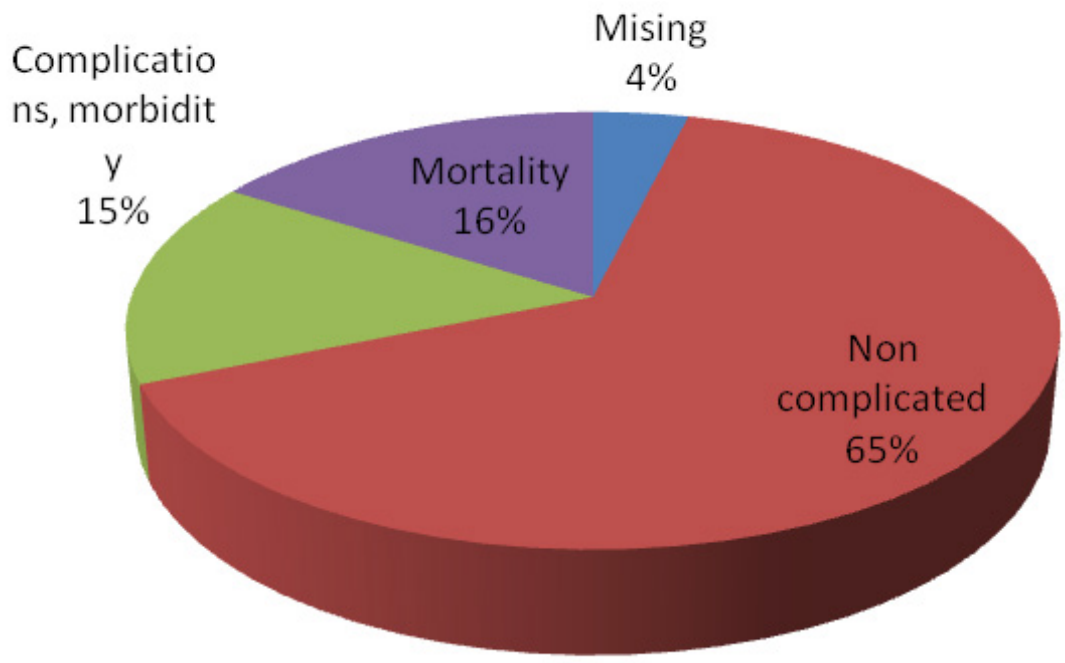

Figure 11: The reported discharge status of the majority of the patients (65\%) does not show any complications during hospitalization. However, $15 \%$ had complications, and $16 \%$ were dead. 
Additionally, on the subject of the discharge status of our population, $65 \%$ of our patients were stable or totally cured, $16 \%$ have died, and $15 \%$ had complications (Figure 11). Mortality and morbidity rates increase with age, with significant $p$-values of 0.03 and 0.047 respectively; they are not linked to gender, and they are more seen in the exudative type of pleural effusion (Table 4 , Table 5 and Table 6).

Comparatively to other studies, patients with exudative effusions, and primarily those caused by malignancy and infection, have higher mortality rates than patients with transudative effusions: The mortality within one month is $11 \%$ for patients with infectious pleural effusion, $35 \%$ for patients with malignant effusion and $7-14 \%$ for patients with congestive heart failure, liver and kidney failure [12-19]. It was believed that the majority of patients with transudate effusions were milder cases than exudate effusions, with less mortality and morbidity rates, less hospitalization, fewer requirements of thoracentesis, and they were treated more easily than exudate effusions (treatment is based on medications rather than invasive or advanced options).

In brief, these results will open up a new discussion and debate in the future to enhance our health care system, and control the risk of complications and mortality attributable to pleural effusion.

\section{Study impact}

This study is the largest study about etiology of pleural effusion in Lebanon, which involves inpatients from three major university hospitals in Beirut: Lebanese Hospital Geitaoui, Rafik Hariri University Hospital and Makassed General Hospital. As well, it's not limited in the determination of the etiology of pleural effusion (exudates versus transudate). It also reports a wide association between different variables: Age, gender, past medical history of each patient, treatment used and discharge status (morbidity and mortality). Furthermore, our results are close to those found in our regional countries (Kuwait, Iran, and Qatar) where tuberculous, parapneumonic, and malignant effusions are the most common types. This is compatible with the global hypothesis which states that in the developing countries like Lebanon, exudate effusions are more reported than transudate ones.

\section{Study limitations}

Some limitations can be noted in our study. First, this study was hospital based rather than population based and performed over one-year period only, which can limit our statistical results. Second, our sample didn't include inpatients that didn't do a thoracentesis. Third, during data collection, some information about different variables were missed, which might affect our result analysis. This problem is possibly related to a weak data processing system in our hospitals, which must be cor- rected in order to improve the medical research results in future.

\section{Conclusion}

In summary, pleural effusion is a common disease confronting the physicians, and knowing its most common etiologies will help to improve the therapeutic options.

In our study, we found that exudative effusion is the most common cause of pleural effusion (78\%), with infectious parapneumonic as the most common exudative cause $(48.8 \%)$.

In comparison with studies performed in developed and developing countries, Lebanon would be classified with developing countries.

It's important to do further studies on a larger population including patients who do not need thoracentesis, and on non-hospitalized patients with pleural effusion, in order to help physicians in the management of this disease.

During our study, we noted that antibiotics were profusely prescribed without obvious indications, more studies on the management of pleural effusion, and the misuse of antibiotics, are needed in our country.

Finally, there is no clear explanation for the increased rate of tuberculosis in Lebanon, further studies on the health status of the immigrants and refugees, could answer many of our questions.

\section{Dedication}

In the hope that this work contributes to more scientific research, we dedicate this thesis to our beloved colleagues, our patients, and families.

\section{Acknowledgments}

We owe our gratitude to the thesis committee, especially Pr. Mirna Chahine, and the jury members for accepting to review our work. Our deepest gratitude for our thesis director Dr. Loubna Tayyara, for her support. We are very pleased and honoured to be students of the Lebanese University, especially the Faculty of Medical Sciences. And last but not least, we would like to thank our family, friends, and hospital directors who made this study happen.

\section{References}

1. Porcel JM (2013) Diagnosis of pleural effusions, Hospital medicine clinics. Gastroentorology 2: 337-357.

2. Mocelin H, Fischer G (2002) Epidemiology, presentation and treatment of pleural effusion. Pediatric Respiratory Reviews 3: 292-297.

3. Miserocchi G (2009) Mechanisms controlling the volume of pleural fluid and extravascular lung water. Eur Respir Rev 18: 244-252.

4. (2019) Pleural effusion, USA: Vanderbilt University Medical Center. 
5. Hooper C, Lee YC, Maskell N, BTS Pleural Guideline Group (2010) Investigation of a unilateral pleural effusion in adults: British Thoracic Society pleural disease guideline 2010. Thorax 65: 4-17.

6. Light $\mathrm{R}$ (2006) Parapneumonic effusions and empyema. Proceedings of the American Thoracic Society 3: 75-80.

7. Light RW, Girard WM, Jenkinson SG, George RB (1980) Parapneumonic effusions. Am J Med 69: 507-512.

8. Light RW (2011) Pleural effusions. MCNA 95: 1055-1070.

9. Kalaajieh WK (2001) Etiology of exudative pleural effusions in adults in North Lebanon. Can Respir J 8: 93-97.

10. Khan F, Alsamawi M, Yassin M, Ibrahim A, Hamza M, et al. (2011) Etiology of pleural effusion among adults in the state of Qatar: A 1-year hospital-based study, East Mediterr Health J 17: 611-618.

11. Porcel JM (2010) Pleural effusion from congestive heart failure. Semin Respir Crit Care Med 31: 689-697.

12. Kookoolis AS, Puchalski JT, Murphy TE, Araujo KLB, Pisani MA (2014) Mortality of Hospitalized patient with pleural effusions. J Pulm Respir Med 4: 184-196.
13. Parikh P, Odhwani J, Ganagajalia C (2016) Study of 100 cases of pleural effusion with reference to diagnostic approach. IJAM 3: 328-331.

14. Heidari B, Bijani K, Eissazadeh M, Heidari P (2007) Exudative pleural effusion: Effectiveness of pleural fluid analysis and pleural biopsy. EMHJ 13: 765-773.

15. Das DK (2015) Age and sex distribution in malignant and tuberculous serous effusions: A study of 127 patients and review of the literature. Geriatric Gerontol Int 15: 11431150.

16. Porcel JM, Light R (2006) Diagnostic approach to pleural effusion in adults. AFPJ 73: 1211-1220.

17. Jany B, Welte $T$ (2019) Pleural effusion in adults-etiology, diagnosis, treatment. Dtsch Ärztebl Int 116: 377-386.

18. Mocelin HT, Fisher GB (2002) Epidemiology, presentation and treatment of pleural effusion. Pediatr Respir Rev 3: 292-297.

19. De Biasi E, Pisani M, Murphy T, Araujo K, Kookoolis A, et al. (2015) Mortality among patients with pleural effusion undergoing thoracentesis. Eur Respir J 46: 495-502. 